\title{
Germanica
}

\section{Neue Sachlichkeit und Arbeitswelt}

Monde du travail et "Nouvelle Objectivité»

\section{Françoise Muller}

\section{(2) OpenEdition}

Journals

Édition électronique

URL : http://journals.openedition.org/germanica/2382

DOI : 10.4000/germanica.2382

ISSN : 2107-0784

Éditeur

Université de Lille

\section{Édition imprimée}

Date de publication : 31 décembre 1991

Pagination : 55-70

ISSN : 0984-2632

\section{Référence électronique}

Françoise Muller, « Neue Sachlichkeit und Arbeitswelt », Germanica [Online], 9 | 1991, Online erschienen am: 07 Juli 2014, abgerufen am 06 Oktober 2020. URL : http://journals.openedition.org/germanica/ 2382 ; DOI : https://doi.org/10.4000/germanica.2382

Ce document a été généré automatiquement le 6 octobre 2020.

(c) Tous droits réservés 


\title{
Neue Sachlichkeit und Arbeitswelt
}

\author{
Monde du travail et «Nouvelle Objectivité»
}

\author{
Françoise Muller
}

1 «Wirtschaft, Technik, Industrie, alles sehr gefragt, alles sehr modern, wir machen mit, wir sind wirklich nicht rückständig' ${ }^{1}$, so ironisierte Erik Reger 1930 die literarischen Versuche seiner Zeitgenossen, die sich mit einem Thema befaßten, das in den zwanziger und am Anfang der dreißiger Jahre in Mode war ${ }^{2}$.

«Modern» war also damals das Technik-Motiv, aber eigentlich nicht ganz neu, denn es gehörte schon, wie auch die Gestaltung der Produktionssphäre, zur Thematik des Naturalismus, der frühen - und - Arbeiterliteratur des Expressionismus. Welches waren nun die schöpferischen Methoden der Autoren, die die verschiedenen Aspekte der Arbeitswelt aufnehmen und literarisch verarbeiten wollten, in einer Zeit, wo an die Stelle der expressionistischen Technikfeindlichkeit die Hinwendung zum, Sachlichen und die Begeisterung für den technologischen Fortschritt aufgetreten waren? Welches waren ihre programmatischen Aussagen? Inwiefern haben sie Neuland betreten?

3 Viele Autoren der zwanziger Jahre haben sich der Industrie als Kulisse bedient: das Reich der «Schlotbarone» ${ }^{3}$, der «Rauch an der Ruhr» ${ }^{4}$ bildeten einen ungewohnten, manchmal idyllisierten Hintergrund. Vorherrschend sind aber die Produktionen der aus dem Industrieproletariat oder aus dem handwerklichen Mittelstand stammenden «Arbeit er dicht er». Die Arbeiterdichtung, die sich während des ersten Weltkrieges entwickelt hatte und als eine eigenständige literarische Gattung betrachtet werden konnte, erlebte nun ihre Blütezeit in der Weimarer Republik. Verherrlichung und zugleich Dämonisierung der Technik, Messianismus, Mystik der Arbeit, Klage über «das proletarische Schicksal» kennzeichnen ihre Werke, deren Schwerpunkt in der Lyrik und im Sprechchor liegt. Typisch für die Arbeiterdichter ist ihre ambivalente Annäherung an die industrielle Sphäre, die sie doch von innen erlebt haben. Wie ein «Mensch im Eisen» pendelt die von ihnen dargestellte Gestalt des Industriearbeiters zwischen Bejahung und Ablehnung der Welt, die sie gefangen hält, zwischen dem Maschinenhaß des Expressionismus und dem Technikkult der Neuen Sachlichkeit. Ob Sklavenarbeit oder Gottesdienst, der Alltag in der Fabrik wird verklärt, wie auch das emotionale, erotisierte Verhältnis zur Maschine, die abwechselnd als fauchendes Tier 
oder als Geliebte erscheint. Dieses Thema, das in der Literatur und im Film der zwanziger Jahre mehrmals auftritt, wird von den Arbeiterdichtern aus der Perspektive des im Arbeitsprozeß stehenden Industriearbeiters behandelt.

In Max Bartheis Roman: «Das Spiel mit der Puppe» taucht das in den zwanziger Jahren oft gebrauchte Motiv der «Girlmaschine» auf: der Tanz vor zwölf Mädchen (wahrscheinlich «Tillergirls«) auf der Bühne versinnbildlicht zugleich Maschinenrhythmus, Fließbandarbeit und Versöhnung mit der industriellen Welt: «Der zwölffache Mensch vereinfachte sich zu einer wundervoll arbeitenden rhythmischen Maschine, die taktschwer und harmonisch auf der Bühne dröhnte. Das war die Überwindung des Tanzes, die Einordnung in den Rhythmus des Alltags, des Kosmos, war Großstadt, zwanzigstes Jahrhundert, Überwindung der Schwerkraft, Vergeistigung und Nutzbarmachung der Materie» (...) So würden einmal die Maschinen sein, kühle, behirnte, eiserne Tiere, ergeben und dienstbar. Der Mensch würde sie ganz beherrschen» ${ }^{5}$.

Während aber der Arbeiterdichter die gewünschte Harmonie als Traumbild oder als Perspektive für die Zukunft auffaßt, behauptet der Journalist Heinrich Hauser in einem nüchternen Zeitungsbericht und zwar "gegen die Literaten», "daß ein Gegensatz zwischen Mensch und Maschinen nicht existiert» und daß «die Maschinen eine Seele haben». Die Artikelerie, die im Februar und März 1928 unter dem Titel «Umgang mit Maschinen» in der «Frankfurter Zeitung» erschien, enthält eine peinlich genaue Beschreibung von Maschinen und Automobilen. Eine metallene Welt wird da hervorgerufen, wo die Menschen, die diese «Meisterstücke der Technik» bedienen, kaum erwähnt werden. Die Maschinen sind es, die mit anthropomorphisierenden Metaphern geschmückt, wie Menschen aussehen: «Die alten Rennwagen sahen sehr menschlich aus: wie alte Generäle, klapprig, steif und bedeckt mit Orden». Heinrich Hauser, der sich für einen Anhänger des Taylorsystems erklärt, schildert 1931 fasziniert das Fließbandsystem bei Ford in «Feldwege nach Chicago»: «So entsteht Harmonie, Rhythmus, eine Art Kosmos $»^{6}$. Im Vergleich zu dieser irrationalen Hingabe wirkt die trockene, präzise Lyrik eines Hannes Küpper auch ein Lobgesang auf die Maschine und die Geschwindigkeit fast ernüchternd ${ }^{7}$. Über diesen ans Lächerliche grenzenden Technikrausch schrieb Brecht sein satirisches Gedicht: «700 Intellektuelle beten einen Öltank an».

6 Den kommunistischen Autoren des 1928 gegründeten «Bundes der proletarischrevolutionären Schriftsteller» (BPRS) geht es weniger um die Wunder der Technik, als um ihre sozialen Auswirkungen.

7 In ihrer Zeitschrift «Die Linkskurve» polemisieren sie gegen die «bürgerlichen Literaten», aber auch gegen die "Arbeiterdichter», die sie als «Kulturapostel» und «Feierabendlyriker» bezeichnen, und werfen ihnen die «Verfälschung des Inhalts durch die Form $»^{8}$ vor. Die Vorbilder der Arbeiterdichter sind Walt Whitman und Dehmel, ihre literarischen Formen sind der Romantik und dem Expressionismus entlehnt; ihnen fehlt aber der «sprachliche Mut», die «experimentelle Kühnheit» der Expressionisten. Obwohl viele von ihnen -wie zum Beispiel der Kesselschmied Heinrich Lersch-imstande sind, den Arbeitsprozeß wirklichkeitsgetreu und mit aller handwerklichen Kenntnis zu beschreiben, gebrauchen sie doch eine mythologisierende Sprache, die jede Aufklärung über die Produktionsverhältnisse unmöglich macht.

8 Tatsachen verlangen also die Mitglieder des BPRS und dokumentarisches Material über die Produktionssphäre. Die «Linkskurve» ruft zum «Marsch auf die Betriebe» 
(Dezember 1929), zum «Marsch auf die Fabriken» (Juli 1930) auf; «Das Gesicht dem Betrieb zu» heißt die Parole (Januar 1930), die Arbeiterkorrespondenz entwickelt sich: 1930 werden 946 Arbeiterkorrespondenzen in der «Roten Fahne» veröffentlicht. Diese Tatsachenberichte geben einen Einblick in die Verhältnisse der Betriebe, dienen gleichzeitig auch zur Schulung neuer Schriftsteller aus den Reihen des Proletrariats. Aus der Arbeiterkorrespondenz gehen zum Beispiel Autoren wie Bredel, Grünberg oder Marchwitza hervor. Die Grenzen der Arbeiterkorrespondenz, die in ihren Anfängen als Literatur aus dem Betrieb überschätzt wurde, werden bald erkannt: «Die Betriebsberichte sind noch keine proletarische Literatur» heißt es in der «Linkskurve» im März 1930. Aber eine Literatur des proletarischen Alltags wird ständig gefördert: Hans Lorbeer mit seinen Chemie-Erzählungen zum Beispiel, Ludwig Turek mit seiner Autobiographie «Ein Prolet erzählt», Hans Marchwitza mit «Schlacht vor Kohle» oder «Walzwerk» oder noch Willy Bredel mit «Maschinenfabrik N. und K.» schildern den Alltag des Industriearbeiters, die Handgriffe an der Werkbank, das Fließband, die Unfälle, die Streikbewegungen und berufen sich dabei meistens auf ihre eigenen Erfahrungen. Der Industriebetrieb wird von der Arbeitsstätte aus betrachtet. Diese Tatsachenliteratur ist aber zugleich eine Parteiliteratur, die sich bewußt von den anderen literarischen Bewegungen absondert. Die Angriffe gegen die Arbeiterdichter, die zum größten Teil der SPD nahestehen, sind nicht nur gegen ihre veralteten literarischen Formen gerichtet, sondern gegen die sogenannten «Klassenversöhnungspoeten» der Sozialdemokratie. Die Tatsachenliteratur der proletarischen Autoren ist nämlich kein neutrales Gebiet, sie hat eine funktionnelle Bedeutung, folgt den Richtlinien der Partei und ihren «Wendungen». Wenn ihre Vertreter die inneren Mechanismen der Arbeitswelt und der Gesellschaft, «erkennen, analysieren, röntgen» wollen - so Bredel ${ }^{10}$ - dann sollen die erworbenen Kenntnisse gleich zur politischen Orientierung des Lesers beitragen. Über die Arbeit s weit schreiben bedeutet nicht nur literarisches Neuland betreten, sondern die Eroberung der Betriebe durch die Arbeiterklasse vorbereiten.

«Neue Themen und abgenutzte Literatur»: so faßt ungefähr Erik Reger in einem 1931 erschienenen Artikel seinen Gesamteindruck über die Werke der proletarischrevolutionären Autoren zusammen: die Parteiliteratur lehnt er ab, weil sie ihr Ziel verfehlt und bei aller Sachlichkeit realitätsfern bleibt: «Wären die Kapitalisten und die Machtorgane so einfältig brutal und so sadistisch wie sie in den sogenannten proletarischen Romanen geschildert worden, so wären sie längst erledigt. Diese Schilderungen verhalten sich zur schwierigen Realität ungefähr so wie die an Drähten gezogenen Ziele auf Schießübungsplätzen sich zur faktischen Beweglichkeit lebender Heere verhalten ${ }^{11} »$.

10 Aber auch gegen die Arbeiter dichter, diese «Barden der Rauchfahnen», «diese Rhapsoden der Schwefelgase» führt Reger einen erbitterten, wenn auch oft humorvollen Kampf. Grotesk ist ihr Gebrauch überholter Stilmittel, grotesk die «Maschinenromantik»: «Eichendorff als Mechanikus. Es schienen so goldendie Sterne, am Fenster ich einsam stand und hörte aus weiter Ferne Maschinenlärm im Land» ${ }^{12}$. «Es ist darum grotesk», schreibt Reger, «weil sie (die Dichter) sich in der Behandlung des Themas von der naiven Empfindung beherrschen lassen, daß die Industrialisierung der Welt das Gefühl für die schönen Künste zerstöre» ${ }^{13}$. «Eine Präzisionästhetik», die «Klarheit und Sauberkeit» verlangt, soll also nach Reger die überholte «Ästhetik der Schornsteine» ${ }^{14}$ ersetzen. Auch die psychologisierenden Produktionen sind in der industriellen Gegenwart fehl am Platze. In seinem programmatischen Aufsatz «Die 
publizistische Funktion der Dichtung», der im März 1931 im «Dortmunder GegeralAnzeiger» veröffentlicht wurde, bekennt er sich zum Übergang «vom Poeten zum Publizisten», «vom privaten Stoff zum öffentlichen Thema». Literatur und Kunst sollen eine aufklärerische Rolle spielen. «Wenn der Künstler überhaupt noch eine Funktion hat, so kann es nur eine publizistische, keine poetische $\operatorname{sein}^{15}$ ».

Die Mode der Reportage, die sich in den zwanziger Jahren durchsetzt, scheint ein Beweis dafür zu sein, daß sich diese Versachlichung der Literatur bereits vollzogen hat, aber selbst die Industriereportagen haben nicht immer mit der «poetischen» Tradition gebrochen.

12 Egon Erwin Kisch, dessen Bestseller «Der rasende Reporter» nicht wenig dazu beigetragen hat, diese Darstellungsart literaturfähig zu machen, schreibt 1924 in seinem Vorwort: «Nichts ist verblüffender als die einfache Wahrheit, nichts ist exotischer als unsere Umwelt, nichts ist phantasievoller als die Sachlichkeit» ${ }^{16} . \mathrm{Zu}$ dieser Exotik der Umwelt gehört für viele die Welt der Industriearbeit und so wurde das Industriegebiet im Westen Deutschlands, das als «Waffenschmiede des Reiches» während des ersten Weltkrieges, dann als «wirtschaftliches Herz Deutschlands», als Reich der «Schlotbarone» und als «Heimat des Proletariats» eine fast mythische Bedeutung bekommen hatte, ein«beliebtes Ausflugsziel der Reporter». Schon Leo Lania hatte in einer 1923 in der «Weltbühne» erschienenen Reportage auf die einzigartige Schönheit der Nachtlandschaft - fast im Stile der Arbeiterdichter - hingewiesen: «Ein phantastisches Bild: wie riesige Wachtfeuer züngeln ringsum rote Flammen aus der Finsternis und drüben im Westen ist der schwarze Nachthimmel in einen grellen, blutigen Schein getaucht (...) ein Gewirr von Hebeln, Stangen, Traversen wird sichtbar nein, es ist nicht der Krieg, es ist die Arbeit, die hier ihr nächtliches Fest begeht» ${ }^{17}$. Dann geht Lania zur konkreten Analyse der politischsozialen Situation über. Dieser Bericht, wo Verklärung und trockene Wiedergabe der Fakten aufeinander folgen, reflektiert das Nebeneinander von Faszination und Tatsächlichkeit, das viele Industriereportagen charakterisiert.

13 Auch Egon Erwin Kisch hat die widersprüchlichen Eindrücke erwähnt, die «uns bewußte Laien» verwirren: «Verstört ist man von den vielen Wundern», schreibt er in seiner Reportage über «Ein Stahlwerk in Bochum». Vom grandiosen Anblick gefesselt, ist der Besucher geneigt, die negativen Vorgänge zu übersehen, auch wenn in seiner Nähe ein Unfall soeben noch mit knapper Not vermieden wurde: «Aber selbst die Erschütterungen solcher Zwischenfälle sind in ihrer Intensität nichts gegen den Eindruck zauberischer Vorgänge, deren staunender Zeuge man ist». Bei aller Größe des Schauspiels versucht er die wirklichen Akteure nicht zu vergessen. Überwältigend sind die Eindrücke, die er in der Gießerei sammelt: «Das Roheisen fließt (...) wie ein flammender Bach in eine Riesenpfanne, auf dem Weg kaskadenfröhlich in Tausende silberne Tröpfchen zerstäubend und den Winterrock des allzu nahe herantretenden Beschauers mit Silber flitter besäend $\aleph^{18}$. Auf diese bewußt ästhetisierende Darstellung des technischen Vorgangs folgt eine Reihe von scheinbar naiven, desillusionierenden rhetorischen Fragen: «Wird nicht auch die Lunge der Arbeiter hier mit diesem eisernen Konfetti überschüttet? Ist er unempfindlich gegen den Schwefeldampf, den uns eben ein Windstoß in die Nase geblasen hat, daß wir husten und tränen müssen? Ist das Gichtgas für ihn kein Gift? $\gg^{19}$ Darauf umreißt er mit wenigen Worten das, was man sich unter «proletarischem Schicksal» vorstellen kann: «In drei Schichten arbeiten die Leute am Hochofen, der jahraus, jahrein, Tag und Nacht nicht erlöschen darf, und an 
jedem dritten Sonntag haben sie sechzehn Stunden Dienst. Vom vierzehnten Lebensjahr an bis zum Tode, der vielleicht schon kommt, während sich andere noch mit dem Studium «abplagen» ${ }^{20}$. Mit derselben leisen Ironie beschreibt er die Stadt Essen, «Das Nest der Kanonenkönige», das Imperium der Familie Kr. (= Krupp): «Kr.Friedhof, Kr.-Lazarett, Kr.-Verwaltungsgebäude, Kr.- Konsumverein, Kr.-Denkmal, Kr. Kr. und wieder $\left.\mathrm{Kr}_{-}\right\rangle^{21}$ ein Thema, das Larissa Reißner ein Jahr später in der «Weltbühne» mit mehr Pathos behandelte ${ }^{22}$.

Als Prototyp der auf Fakten beruhenden, aber ästhetisierendem Reportage kann dagegen Heinrich Hausers «Schwarzes Revier» gelten, das 1930 beim Fischer Verlag erschienen ist. «Nichts ist geschrieben worden, was nicht gesehen oder erlebt ist», heißt es im Vorwort, aber der Autor betrachtet die Ruhrprovinz mit Augen, die das «Wundern» nicht verlernt haben und die Hochöfen, die er zu sehen bekommt, sind "von einer unbeschreiblichen Eleganz», sogar die Silhouetten der Zechen, der Hüttenwerke, der Kokereien werden als dekorative Muster empfunden: «Einmal vertraut geworden mit den Elementen dieser Landschaft, begegnet man in dem scheinbar so ungeheuer verschiedenartigen Bild immer wieder den gleichen Farben und den gleichen Umrissen, so wie das Ornament einer Tapete wiederkehrt» ${ }^{23}$. Die Industriearbeit wird durchgängig mit einem natürlichen Vorgang gleichgestellt: Von der «Zeugung des Stahls» ist die Rede, der in der Fabrik «geboren wird». Anläßlich einer Besichtigung der Krupp-Werke werden sogar die germanischen Heldensagen bemüht: «Es ist ein langer Weg, der von Wieland dem Schmied zu Krupp in Essen führt» ${ }^{24}$. Die Arbeiter werden meistens als Statisten dargestellt oder als Elementarwesen, die in dem Schacht kriechen. Sätze wie «Unbegreiflich dem Verstand ist der geheimnisvolle Trieb, der Menschen über Meere und unter die Erde treibt» ${ }^{25}$, machen die totale Blindheit des Autors den gesellschaftlichen Verhältnissen gegenüber vollends deutlich. Außerdem reflektieren seine «unpolitischen Aufzeichnungen» die Ideologie der Werkgemeinschaft. Es handelt sich hier offensichtlich um eine Verfälschung durch die Form: sein journalistischer Tatsachenbericht - «mit fotographischen Aufnahmen des Verfassers» illustriert - weist in der Tat viele Züge der Arbeiterdichtung auf.

Nicht «Schwarzes Revier», sondern «Kohlenpott» - ein Wort der derben Kumpelsprache -, heißt die Reportage von Georg Schwarz, die 1931 bei der Büchergilde Gutenberg erschien und 1986 in der Reihe «Ruhrland-Dokumente» neu herausgegeben worden ist. Georg Schwarz ist in Dortmund geboren und distanziert sich gleich von den vielen Reportern, die - oft von der Hauptstadt - in seine Heimat kommen, um den Pulsschlag der deutschen Wirtschaft zu fühlen und dabei eine "phantastische, fremde Welt» zu entdecken, von der sie aber nur das Äußere, die Kulisse sehen: «Die durchreisenden Reporter schmecken dabei ordentlichen Ruß auf der Zunge und lassen ihn genießerisch wie eine Prise Kaviar zergehen. Das ist kerniger als das Nuttenparfüm Berliner Ballsäle. Es füllt die Zeilen, die Ästhetik der Industriebauten zu preisen, und es spart einem die Mühe, langweilige Zahlen von Arbeitslöhnen, Abschreibungen und Dividenden $\mathrm{zu}$ vergleichen ${ }^{26}$. Schwarz liefert ein umfangreiches dokumentarisches Material, ohne Ästhetisierung aber ohne die Trockenheit des Tatsachenberichts. Er entwirft ein Panorama des Ruhrgebiets: Städte, Industriewerke, Landschaft und Menschen, Dichter aus dem Proletariat. Seine linke Tendenz ist unverkennbar: er denunziert die Machenschaften der Arbeitgeber, die «Seelenbewirtschaftung der Industrie» durch Werkzeitungen, das deutsche Institut für Arbeiterschulung (Dinta), das arbeitsphysiologische Institut, wo neue Methoden zur Steigerung der 
Maximalleistung ausgearbeitet werden. Sachlich ist seine Analyse, aber klassenbewußt und sie gehört zum Teil in die Reihe der proletarisch-revolutionären Schriften.

Obwohl diese Reportagen über die Arbeitswelt gemeinsame Züge aufweisen, zeichnet sich keine einheitliche Tendenz ab. Die Reporter kritisieren sich gegenseitig. Georg Schwarz polemisiert gegen die «idealisierenden Poeten und Hymnensänger der Industrie», aber auch gegen die «sachlichen» und «rasenden» Reporter. Über Hausers «Schwarzes Revier» schreibt Reger im «Scheinwerfer» einen regelrechten Verriß. Von Kisch meint er, er begnüge sich mit der «Oberfläche» und von Schwarzens «Kohlenpott» hält er nicht viel: «Leider macht man die betrübliche Feststellung, da der Schwarzsehe Kohlenpott bis zum Rande gefüllt ist mit Binsenwahrheiten und dem statistischen Material, das die Presseämter geben ${ }^{27}$ ».

Auch die Fotografien, die die Reportage begleiten, sind Gegenstand der Kritik: Schwarz, der seine eigene Reportage mit Aufnahmen des Arbeiterdichters Erich Grisar illustriert hat, nörgelt an den Produktionen der sensationslustigen «für alle Schönheiten der Welt empfänglichen Schornstein-schräg-nach oben Photographen $»^{28}$. Regers Position scheint in dieser Hinsicht ziemlich rückständig zu sein, denn er sieht in den phototechnischen Qualitäten der Illustrationen eine Gefahr, insofern als die Kamera kein «ergänzendes oder unterstreichendes, sondern ein Ersatzinstrument» zu werden droht: Als Beweis dafür gilt Hausers Reportage «Schwarzes Revier», von dem der Autor selbst sagt, es sei als ein «lockeres Gewebe» aufzufassen, als eine laterna magica: Bilder auf eine Leinwand geworfen, während der Autor daneben hastige und ziemlich ungeschickte Erklärungen dazu abgebe. Nach Reger besteht also die Gefahr, daß der Text zugunsten der Aufnahmen vernachlässigt wird, daß der Kampf um das Wort aufgegeben wird. Damit wäre die Reportage als literarische Gattung bedroht: «Zur Sache also», schreibt Reger im Jahre 1930, «das heißt - zum Wort! Schluß mit den Bilderbüchern, Rückkehr zur Darstellung durch das Wort! $»^{29}$.

Die Autoren sind sich also weder über den Inhalt noch über die Methodik einig; jeder besteht auf seine Anschauungs- und Gestaltungsweise, was Kracauer bekannte Aussage bestätigt: «Hundert Berichte aus der Fabrik lassen sich nicht zur Wirklichkeit der Fabrik addieren, sondern bleiben bis in alle Ewigkeit hundert Fabrikansichten. Die Wirklichkeit ist eine Konstruktion. Gewiß muß das Leben beobachtet werden, damit sie erstehe. Keineswegs ist sie jedoch in der mehr oder weniger zufälligen Beobachtungsfolge der Reportage enthalten, vielleicht steckt sie einzig und allein in dem Mosaik, das aus den einzelnen Beobachtungen auf Grund der Erkenntnis ihres Gehalts zusammengestiftet wird. Die Reportage Photographien das Leben; ein solches Mosaik wäre sein Bild ${ }^{30}$ ».

19 Auch Lukacs' Stellung zur Reportage-Literatur ist bekannt. Seine kritische Beurteilung von Bredels Betriebsromanen («Maschinenfabrik N. und K.» und «Rosenhof Straße») und von Ottwalts Justizroman «Und sie wissen was sie tun» in der «Linkskurve» gibt ihm Anlaß, sich in einer Reihe von Artikeln über die neuen schöpferischen Methoden der Tatsachenliteratur auszusprechen; Bredels Romane sind bloß Entwürfe eines Romans, die Personen sind scharf umrissene Charakterrollen, die Sprache ist die des Presseberichts oder des Gewerkschaftsprotokolls. Ottwalts Roman entlarvt nur Einzelheiten und nicht die wahren Mechanismen des Justizwesens. Nicht die Reportage stellt Lukacs in Frage, sondern den Versuch einer Erneuerung des Romans durch die Mittel der Publizistik. Die Totalität, wonach der Roman streben soll, «ist nicht die Summe der Tatsachen». So läßt er zum Beispiel in dem Aufsatz, den er im Dezember 
1932 in der letzten Nummer der «Linkskurve» veröffentlichte, Gorki sprechen: «Ich (Gorki) habe die Ausbeutung und die Unterdrückung der russischen Arbeiter, sowie ihre beginnende Auflehnung gegen sie in meinem Roman «Die Mutter» «herkömmlich» gestaltet und hielt es für gänzlich überflüssig, die Fabrikanten, den Aufsichtsrat, den Grundbesitzer, den Minister des Innern, den Zaren einzubeziehen»" ${ }^{31}$.

Und doch gab es in dieser Zeit Autoren, die versucht haben, die herkömmlichen Romanformen zu sprengen. Zwei «neusachliche» Industrieromane sollen als Beispiel genommen werden; einer ist leider um diese Zeit ungedruckt geblieben, der Verfasser war Franz Jung, der andere ist mit dem Kleistpreis ausgezeichnet worden, sein Autor war Erik Reger.

21 Anläßlich einer Kritik von Arnolt Bronnens Oberschlesienroman «O.S», weist Reger auf den Mangel an Tatsachensinn des Autors, der die Triebkräfte der Kämpfe zwischen Deutschen und Polen nach dem ersten Weltkrieg und die «realen Mächte», das heißt, die wirtschaftlichen Zusammenhänge, die diese Kämpfe bestimmten, außer acht läßt: «Nicht nur Ideale waren im Spiele, sondern auch ungefähr 67 Kohlengruben, 16 Zinkund Bleigruben, 22 Zinkhütten und Schwefelsäurefabriken, 25 Stahlwerke, 14 Walzwerke und dazu pro Jahr 2,5 Millionen Tonnen Koks und eine Million Tonnen Roheisen ${ }^{32}$ ». Der oberschlesische Roman, den sich Reger damals wünscht, ist schon zwei Jahre vorher von Franz Jung geschrieben und seitdem von mehreren Verlagen abgelehnt worden: So lautet der Projektvorschlag, den Jung 1926 der Büchergilde Gutenberg unterbreitet hatte: «Geschichte eines Industriereviers mit Wirtschaftskämpfen, Trustenentwicklung, Krisen etc als Rahmen, und dahineingewoben naturalistisch geschildert, Lebensentwicklung des Arbeiters, der Familie, seine Stellung zu allen heutigen Fragen insbesondere der kulturellen». Die Büchergilde Gutenberg schickte aber das Manuskript zurück, wie auch andere linke Verlage. Erst im Jahre 1987 ist dieses Buch unter dem Titel «Gequältes Volk» endlich herausgegeben worden $^{33}$. Franz Jung, der durch seine expressionistische und dadaistische Prosa und seine linksradikalen Werke hervorgetreten ist, lebt um diese Zeit seit 1923 in Berlin, wo er eine Reihe von Wirtschaftskorrespondenzen und einen Fotodienst für die Wirtschaftspresse gegründet hat. Nach mehreren Theaterexperimenten bei der Piscatorbühne bedeutet ihm dieser Roman ein weiterer Versuch «in die Literatur zurückzukehren». Dabei distanziert er sich aber auch von seinen früheren literarischen Positionen, die «in den Strudel der Fraktionskämpfe und Parteiauseinandersetzungen» geraten waren, und von der Produktion seiner sogenannten «roten Jahre». Eine Einladung, dem BPRS beizutreten, läßt er unbeantwortet. Nun in diesen späten zwanziger Jahren, die er selbst als «die grauen Jahre» bezeichnet hat, hat er «für die Fragestellung der Beziehungen der Menschen untereinander allerdings einen breiteren und vor allem gefestigteren Blickkreis gewonnen ${ }^{34} »$. Die Quellen zu seiner Arbeit findet er in seinen eigenen Erfahrungen er ist ein gebürtiger Oberschlesier - und in dem umfangreichen Material, über das er als Wirtschaftsjournalist verfügt. Und in der Tat wird die Gesellschafts- und Wirtschaftskrise, die seine Heimat nach dem ersten Weltkrieg erschütterte, in ihrer ganzen Komplexität dargestellt. Der Roman bietet eine Zusammenfassung der wichtigsten Faktoren, die im Spiele waren, ein buntes Durcheinander der vielschichtigen Zusammenhänge: Rohstoffmärkte, Kapitalverschiebungen, die eine Konsequenz der Teilung Oberschlesiens waren, Interessenkonflikte und Abmachungen der deutschen und polnischen Konzerne, ihre Auswirkungen auf die Arbeiterschaft, Streiks und Krawalle, die ambivalente Rolle der Kirche und der Gewerkschaftsführer 
und schließlich die Abwanderung der Arbeitslosen. Über die Geschehnisse wird nüchtern und distanziert berichtet, nur im letzten Kapitel, «Kehraus» überschreitet Jungdas Gesetz der Objektivität und geht sogar zur Ich-Form über: «Land meiner Heimat sei gesegnet! Land meiner Heimat sei verflucht!» Er fällt schließlich aus der Rolle des sich neutral gebenden Beobachters und seine Parteinahme für die proletarischen Opfer der Krise kommt deutlich hervor: «Der so lange künstlich aufrechterhaltene Trennungsstrich als Deutsche oder Pole war mit einem Male verwischt. Wieder wurden sie ein Volk, ein einheitliches Volk, das oberschlesische Volk, das ohne Arbeit war und Brot und das in seinen besten Teilen ausgesiedelt werden sollte (...) Unter dem Schutz dieser Bewegung bereiteten die oberschlesischen Industriellen die Umstellung ihrer Betriebe vor. Sie kehrten aus und gründlichst ${ }^{35}$ ». Das letzte Kapitel schließt sich merkwürdigerweise an die linksradikalen Schriften seiner «roten Jahre» an.

Jungs «neusachlicher» Roman hat sich 1927 im Literaturbetrieb nicht durchgesetzt. Vielleicht lag es an dem ungewohnten Thema: erst einige Jahre später florierte die eigentlich meistens nationalistisch gefärbte und antipolnische Oberschlesienliteratur.

«Gequältes Volk» ist ein bunt zusammengewürfeltes, loses Gebilde, ein Durcheinander von Fakten und Fiktion, in dem die verschiedenartigsten Erzählformen und Stilmittel (Geschichtsschreibung, Anekdote, naturalistische Schilderung, Methoden des Detektivromans, langatmige wirtschaftswissenschaftliche Abhandlungen) nebeneinander bestehen. Einige Kapitel wie «O Täler weit, O Höhen! », über die Zerstörung durch die Industrie der Eichendorffschen Romantik und das Waldsterben, ein damals neues Thema, oder "Der Wurm», das die Rationalisierung eines Gaswerkes thematisiert, können als kleine, in sich geschlossene Novellen betrachtet werden. Nahtstellen fehlen zwischen den inhaltlichen und stilistischen Elementen. Diese Methode der Montage-Technik mit Dokumentation und wissenschaftlichen Erörterungen hatte Jung in seinen Werken der frühen zwanziger Jahre schon angewandt wie zum Beispiel in «Die Eroberung der Maschinen». Neu war aber das, was er selbst die «voraussetzungslose Beobachtung» der Zustände nannte.

Ohne Anteilnahme und ohne Kommentar werden die verschiedenen Episoden von Erik Regers Roman «Union der festen $\mathrm{Hand}^{36}$ » dargestellt: sie sollen für sich selbst sprechen. Als Angestellter des Krupp-Konzerns, wo er von Kriegsende bis 1927 als Buchhalter und Pressesprecher arbeitete, hat Erik Reger Einblick in die Realität des Betriebs bekommen. Nun versucht er seinen eigenen literarischen Ansprüchen gerecht $\mathrm{zu}$ werden und gestaltet am Beispiel der Kruppwerke die Geschichte des Ruhrgebiets von 1918 bis zur Wirtschaftskrise von 1929. Er schildert die Rivalitäten der Großindustriellen, die Verflechtungen der Konzerne, ihre Allianz mit der politischen Reaktion und schließlich mit dem Nationalsozialismus. Er deckt die Praktiken der Machthaber auf, die die Konfrontation mit den Arbeitern zugunsten der «weichen Methode», das heißt der Beeinflussung der Massen, aufgeben, enthüllt die geheime Rolle der Pressebüros der Werkzeitungen und ihre geschickte Propagandaarbeit und zeigt, wie die vielen Vereine und sogar die Wohlfahrtseinrichtungen zur Manipulation der Arbeiterschaft beitragen. Zur Verarbeitung dieses umfangreichen Materials entschied sich Erik Reger für die Fiktion. Die Orte, die Personen, die Institutionen, die für Eingeweihte leicht identifizierbar sind, tragen fiktive Namen (Krupp $=$ RischZander) (Reichsverband der deutschen Industrie = Union der festen Hand). Wie in den proletarisch-revolutionären Romanen haben bei Reger die proletarischen 
Heldengestalten eine exemplarische Funktion, was einer gewissen Vereinfachung, einer Typisierung entspricht. Sie vertreten die verschiedenen Haltungen der Arbeiterklasse: den Radikalen, den Sozialisten, den Opportunisten und ... den Arbeiterdichter (wahrscheinlich Christoph Wieprecht), der eine Firmenhymne schreibt: «Symphonie der Arbeit». Die Arbeiterklasse wird nicht idealisiert, manchmal sogar ziemlich abschätzig betrachtet: «Wurde die Tür aufgemacht, so schlug ein Brodem heraus, wie aus einem Kanincbenstall ${ }^{37}$ ». Der proletarische Hauptheld, ein Kranführer, ist Träger der Desillusion, der Entsagung und Niederlage der Arbeiterklasse von 1918 bis 1929: er ist Spartakist, dann Kommunist, schließlich indifferenter Arbeitsloser. Viele - und vor allem die Mitglieder des BPRS - haben Erik Reger seine «standpunktlose» Haltung zum Vorwurf gemacht: er wollte aber mit dieser Aufklärungsarbeit keiner Partei, keiner Weltanschauung dienen. Aber die zahlreichen Beschreibungen der Arbeitssphäre entsprechen merkwürdigerweise nicht dem, was man von einem Kritiker erwarten könnte, der die Arbeiter dichter so oft und so geistreich parodiert hat: immer wieder greift er nämlich $\mathrm{zu}$ den sogenannten «abgenutzten» Metaphern und bedient sich des Wortschatzes der Arbeiterdichtung. So werden die Maschinen wahrgenommen: «Sie lauern, diese gefräßigen Ungeheuer, sie lauern fauchend ihrer Beute auf ${ }^{38} \%$. Die Arbeiterdichter werden von Ihm abwechselnd verspottet und nachgeahmt: «Das Gehirn, das Gefühl, das Nervensystem dieser Maschine, die für jede Störung ein Heilmittel in sich trug, war vollkommener, als es bei einem Menschen je sein konnte» ${ }^{39}$. Offentsichtlich ist aber seine aufklärerische Absicht: um Tatsachen geht es, die überprüfbar sind und die Literatur wird vor allem nach ihrem Nutzwert gemessen, wie es die «Gebrauchsanweisung», beweist, die dem Roman als Vorwort dient.

Die Autoren der neusachlichen Epoche, die als Vertreter der Industrieliteratur gelten, haben keine einheitliche Gruppe oder Strömung gebildet. Sie versuchten immer wieder, sich als Individuen, oder als «Bund» von den anderen abzugrenzen und schreckten auch nicht vor der Polemik zurück. Sogar Erik Reger, der eigentlich ein Einzelgänger war, schrieb unter mehreren Pseudonymen in der Zeitschrift «Der Scheinwerfer», scheinbar um beim uneingeweihten Leser den Eindruck zu erwecken, daß eine ganze Gruppe von Autoren seine Position vertrat.

Es lassen sich jedoch viele gemeinsame Züge abzeichnen: Abkehr vom Psychologismus und Hinwendung $\mathrm{zu}$ den sozialen Fragen, Gestaltung typischer Figuren mit exemplarischer Funktion, möglichst genaue Beschreibung der Arbeitsvorgänge und Aufdeckung der Mißstände in der Betriebssphäre, Einschätzung der literarischen Formen nach ihrem Nutzwert, durchgängiges Pendeln zwischen Technikrausch und Ernüchterung, und schließlich, bei aller Objektivität, eine ständige Neigung zur Ästhetisierung der Arbeitssphäre, die sogar in einem Standardwerk der Neuen Sachlichkeit wie «Union der festen Hand» zu finden ist. 


\section{NOTES}

1. - Heinz Lamprecht (Erik Reger): «Maschinenwelt und Gartenlaubenromantik», in Der Scheinwerfer April-Mai 1930. Die wichtigsten Artikel aus dem «Scheinwerfer» sind von Erhard Schütz und Jochen Vogt in der Reihe «Ruhrland-Dokumente» (Klartext) unter dem Titel «Der Scheinwerfer -ein Forum der Neuen Sachlichkeit», 1986 neu herausgegeben worden.

2. - Zu diesem Thema siehe Helmut Lethen: «Neue Sachlichkeit 1924-1933», Stuttgart 1970 («Neusachliche Bilder der Produktionssphäre» S.58-92).

3. - Wilhelm Heinrich Dammann : Der Schlotbaron, Stuttgart 1926.

4. - Felix Wilhelm Beielstein : «Rauch an der Ruhr», Stuttgart 1932.

5. - Max Barthel : Das Spiel mit der Puppe, Leipzig, 1926, S. 144-145.

6. - Heinrich Hauser : Feldwege nach Chicago, Berlin, 1931, S. 232.

7. - Hannes Küpper : «327 Stundenkilometer»; «Notwendigkeit», in Der Scheinwerfer, Okt. 1927, Nov. 1927.

8. - Armin Kesser : «Arbeiterlyrik der SPD», in Die Linkskurve, Oktober 1932, S. 17.

9. - N. Kraus : «Gegen den Ökonomismus in der Literatur frage », in Die Linkskurve, März 1930, S. 10.

10. - Willy Bredel : «Einen Schritt weiter», in Die Linkskurve, Januar 1932.

11. - Erik Reger : «Neue Themen und abgenutzte Literatur», in Dortmunder General-A nzeiger, 8.1.31.

12. - Erik Reger : «Maschinen weit und Gartenlaubenromantik», in Der Scheinwerfer, April-Mai 1930.

13. - Fritz Schulte Ten Hoevel (Erik Reger) : «Literatur der komischen Käuze», in Der Scheinwerfer, April 1931.

14. - Walter Enkenbach (Erik Reger) : «Die Erneuerung des Menschen durch den technischen Geist», in Der Scheinwerfer, Oktober 1928.

15. - Erik Reger : «Die publizistische Funktion der Dichtung», in Dortmunder General-Anzeiger, 31 März 1931.

16. - Egon Erwin Kisch : «Der rasende Reporter», Berlin 1925 (Vorwort S. VIII).

17. - Leo Lania: "An der Ruhrfront», in Die Weltbühne, 1923, I, S. 94-97. Zum Thema Ruhrreportage siehe : Erhard Schütz : «Romane der Weimarer Republik» UTB, 1986, S. 125-146.

18. - Egon Erwin Kisch: "Stahlwerk in Bochum, vom Hochofen aus gesehen» in Der rasende Reporter, (1924) (Reclam «Egon Erwin Kischs Reportagen», S. 79).

19. - Ibid., S. 79.

20. - Ibid., S. 79-80.

21. - Egon Erwin Kisch : «Das Nest der Kanonenkönige: Essen», in Der rasende Reporter (Reclam : «Egon Erwin Kischs Reportagen», S. 85-86).

22. - Larissa Reiner : «Krupp und Essen», in Die Weltbühne, 1925, II, S. 729-734.

23. - Heinrich Hauser : «Schwarzes Revier», Fischer Verlag, Berlin, 1930, S. 22.

24. - Ibid., S. 47.

25. - Ibid., S. 38.

26. - Georg Schwarz : «Kohlenpott» (1931); Neuausgabe: «Ruhrland-Dokumente», Klartext, 1986, S. 1.

27. - Ernst Stahlburg (Erik Reger) : «Ein Kohlenpott oder die Erfindung der Grasnarbe», in Der Scheinwerfer.

28. - Georg Schwarz : «Kohlenpott» (1931); Neuausgabe «Ruhrland-Dokumente», Klartext, 1986, S. 36 . 
29. - Fritz Schulte Ten Hoevel (Erik Reger) : «Das dritte Auge des Reporters» in Der Scheinwerfer, Januar, 1930.

30. - Siegfried Kracauer: «Die Angestellten» (Erstveröffentlichung 1929 in der Frankfurter Zeitung, Suhrkamp, S. 16.

31. - Georg Lukacs : «Aus der Not eine Tugend», in Die Linkskurve, Nov.-Dez. 1932, S. 22.

32. - Karl Westhoven (Erik Reger) : «O. S. Landkarte contra Dichter», in Der Scheinwerfer, Okt. 1929.

33. - Franz Jung: «Gequältes Volk», Edition Nautilus, Hamburg, 1987 (Aus dem Nachlaß herausgegeben mit einem Nachwort von Walter Fahnders).

34. - Franz Jung : «Das Erbe» (aus dem «Viermänner-Buch», 1929, erschienen) in Der tolle Nikiaus, Reclam, Leipzig, 1980, S. 234.

35. - Franz Jung : «Gequältes Volk», Hamburg, 1987, S. 144-146.

36. - Erik Reger : «Union der festen Hand», 1931, im Rowohlt Verlag erschienen. Neuauflage Aufbau Verlag, 1946. Neuauflage Scriptor, Verlag, 1978, mit einem Nachwort von Karl Prümm.

37. - Erik Reger : «Union der festen Hand» Scriptor, 1978, S. 226.

38. - Ibid., S. 182.

39. - Ibid., S. 410.

\section{RÉSUMÉS}

In einer Zeit, wo an die Stelle der expressionistischen Technikfeindlichkeit die Begeisterung für den technologischen Fortschritt aufgetreten ist, pendelt noch die Industrieliteratur zwischen der «Maschinenromantik», der «Ästhetik der Schornsteine», und der «Präzisionsästhetik» der neusachlichen Autoren, die die inneren Mechanismen der Arbeitswelt analysieren und «röntgen» wollen. Während die Vertreter der Arbeiterdichtung die Technik abwechselnd verherrlichen und dämonisieren, versuchen die Autoren des «Bundesproletarisch-revolutionärer Schriftsteller» die Tatsachenberichte und «Betriebsromane» für den Klassenkampf auszunutzen. Die neuen Stoffe werden aber meistens noch mit herkömmlichen Mitteln behandelt und selbst die Industriereportage, die um diese Zeit literaturfähig wird und viele ästhetisierende Züge aufweist, bricht nicht immer mit der «poetischen» Tradition. Mit ihren Industrieromanen, in denen die Totalität einer Gesellschaftssituation ins Tageslicht gerückt wird, betreten Franz Jung mit «Gequältes Volk» und vor allem Erik Reger, dessen «Union der festen Hand» ein Standardwerk der neusachlichen Literatur geblieben ist, literarisches Neuland.

À une époque où au refus de la technique propre à l'expressionnisme a succédé le culte de la technologie et du progrès, la «littérature du monde industriel» oscille encore entre le «romantisme de la machine », l'« esthétique des cheminées d'usine » et une esthétique de la précision prônée par les auteurs de la Nouvelle Objectivité qui se proposent d'analyser et de « radiographier » les mécanismes internes du monde du travail. Tandis que les représentants de la «Arbeiterdichtung» glorifient et démonisent tour à tour la technique, les auteurs prolétariensrévolutionnaires utilisent dans le cadre de la lutte des classes les documentaires et les « romans d'entreprise ". Cependant ces thèmes nouveaux sont encore traités le plus souvent à l'aide de procédés littéraires traditionnels et même le reportage sur la sphère industrielle, qui acquiert à cette époque droit de cité dans le monde des lettres, n'échappe pas à l'esthétisation et ne rompt pas avec une tradition dite "poétique ». C'est avec des « romans industriels», dont l'ambition 
était d'embrasser - objectivement - la totalité de la réalité sociale d'une région et d'une période que des auteurs tels que Franz Jung et surtout Erik Reger («Union der festen Hand») ont fait œuvre de novateurs.

\section{AUTEUR}

FRANÇOISE MULLER

Université Charles de Gaulle - Lille III 